Gut, 1984, 25, 405-411

\title{
Comparison of the effects of medium and long chain triglyceride containing liquid meals on gall bladder and small intestinal function in normal man
}

\author{
S D LADAS, P E T ISAACS, G M MURPHY. AND G E SLADEN \\ From the Gastroenterology Unit, Guy's Hospital Medical School, London
}

SUmmary We investigated the effects of medium (MCT) and long (LCT) chain triglyceride test meals on gall bladder contraction (using ultrasonography) and small intestinal bile acid concentrations and ileal flow rates (using intubation techniques) in normal individuals. Comparing the effects of ingesting medium chain triglyceride and long chain triglyceride meals, the gall bladder volume was reduced by $23.4 \pm 33.6 \%$ and $59 \cdot() \pm 17 \cdot 1 \%$ respectively (mean \pm SD, $\mathrm{p}<0 \cdot(01, \mathrm{n}=13)$, the jejunal bile acid concentrations increased significantly only after the ingestion of long chain triglyceride $(\mathrm{p}<0 \cdot 05, \mathrm{n}=5)$, while the volume of the postprandial jejunal contents was not significantly different $(540 \pm 150,522 \pm 169 \mathrm{ml}$, medium chain triglyceride $v s$ long chain triglyceride, $\mathrm{p}=(0 \cdot 2, \mathrm{n}=5)$. The postprandial colonic inflow was $48 \cdot 5 \pm 12 \cdot 5$ and $123 \cdot 4 \pm 35 \cdot 2$ $\mathrm{ml} / \mathrm{h}$ (medium chain triglyceride $v s$ long chain triglyceride, $\mathrm{p}<0 \cdot(01, \mathrm{n}=5$ ). Thus medium chain triglyceride, compared with long chain triglyceride, produces a smaller input of bile acids into the small intestine and a smaller volume of fluid delivered to the colon. These observations may be relevant to the beneficial effects of medium chain triglyceride substitution for long chain triglyceride in the treatment of diarrhoea in patients with small intestinal disease.

In many patients with a reducęd small intestinal mucosal area due to resection or disease, the replacement of dietary long chain triglyceride by medium chain triglyceride in the diet has been shown to reduce both steatorrhoea and diarrhoea, ${ }^{1-}$ 5 as well as faecal electrolyte excretion. ${ }^{1-4}$ These effects are believed to be because of the rapid absorption of medium chain triglyceride, which occurs independently of the micelle formation, intraluminal hydrolysis and mucosal reesterification, ${ }^{6-8}$ and to the absence of the secretory stimulus of unabsorbed long chain fatty acids in the intestinal lumen. ${ }^{+}$Medium chain triglyceride also differs from long chain triglyceride in its effect on bile salt metabolism. This may be of considerable physiological importance because both bile acids and long chain fatty acids interfere with water and electrolyte absorption from the small intestinal lumen. ${ }^{9} 10$

In the rhesus monkey, medium chain triglyceride feeding decreased bile salt synthesis, pool size and

Address for correspondence: Dr P Isaacs. Gastroenterology Unit. Guys Hospital Medical School. St Thomas Street. London SEI 9RT.

Received for publication 18 July 1983 biliary secretion. ${ }^{11}$ There have been no similar long term comparisons made in man, but we have found lower jejunal bile salt concentrations after a medium chain triglyceride meal compared with a long chain triglyceride meal. ${ }^{12}$ This led us to test the hypothesis that the ingestion of medium chain triglyceride rich as compared with long chain triglyceride rich meals might lead to a weaker gall bladder contraction, ${ }^{13}$ resulting in lower luminal bile acid concentration and a lower postprandial ileal flow.

We therefore measured gall bladder contraction using ultrasound and ileal flow and using intestinal perfusion after ingestion of long chain triglyceride rich and medium chain triglyceride rich test meals.

\section{Methods}

SUBJECTS

Eighteen healthy volunteers aged 22-65 years old ( 12 men and six women) ingested the medium chain triglyceride and long chain triglyceride test meals on two separate occasions. In 13 of these subjects, the changes of gall bladder volume were assessed by ultrasound. In the other five subjects, changes in 
luminal bile acid concentrations and jejunal and ileal aqueous volumes were assessed by jejuno-ileal intubations. Written informed consent was obtained from all subjects. The protocol was approved by the Guy's Hospital Committee on Ethical Practice (January 1982). Women of child bearing age were not studied by intubation.

\section{TEST MEALS}

Each meal diluted in tap water had a final volume of $300 \mathrm{ml}$ and provided $1.7 \mathrm{MJ}(400 \mathrm{Kcal})$. Thirty six per cent of the calories were derived from fat, $48 \%$ from carbohydrate and $16 \%$ from protein. In both meals the carbohydrate was a mixture of mono-, oligo- and polysaccharides, and the protein was derived from whey, casein and soy hydrolysates. Eighty per cent $(w / w)$ of the fat in the medium chain triglyceride meal was medium chain triglycerides $\left(\mathrm{C}_{6}-\mathrm{C}_{12}\right)$ compared with only $31 \%(\mathrm{w} / \mathrm{w})$ in the long chain triglyceride meal. The medium chain triglyceride meal had an osmolality of $460 \mathrm{mOsm} / \mathrm{kg}$ and sodium concentration of $60 \mathrm{mmol} / \mathrm{l}$, while those of the long chain triglyceride were $350 \mathrm{mOsm} / \mathrm{kg}$ and $24 \mathrm{mmol} / \mathrm{l}$ respectively. The meals were bland tasting and did not produce symptoms.

GALL BLADDER ULTRASOUND STUDY

After an overnight fast (14 hours) the volunteers drank the contents of a numbered test meal containing either the medium chain triglyceride or the long chain triglyceride test meal. The test was repeated on a second day using the other meal at random order. The gall bladder volume was assessed blindly by one of us (PETI) at time zero, 30, and 60 minutes using a real time ultrasound system (system 285. Diagnostic Sonar Ltd, Livingston, Scotland) equipped with a $128 \mathrm{~mm} 3 \cdot 0 \mathrm{MH}_{z}$ transducer positioned to obtain a scan showing an axial section of the gall bladder at maximum length (L) at each time interval and a single scan across the axis of the gall bladder to obtain the transverse diameter $(w)$ and antero-posterior diameter (D). Assuming that the gall bladder is cylindrical, the volume was calculated as $\mathrm{V}=\pi \mathrm{L}[(\mathrm{w} / 2) \times(\mathrm{D} / 2)] .^{14-16}$ This method tends to reproducibly overestimate the absolute gall bladder volume, ${ }^{15}$ but should show changes in response to different meals.(Fig. 1).

JEJUNO-ILEAL INTUBATION STUDIES

After an overnight fast, the volunteers swallowed a weighted polyvinyl radio-opaque three lumen tube which was positioned so that its aspiration ports were located 10 to $30 \mathrm{~cm}$ beyond the ligament of Treitz and at the terminal ileum (mercury bag in the caecum). Twenty four hours were necessary for the tube to reach the desired position which was

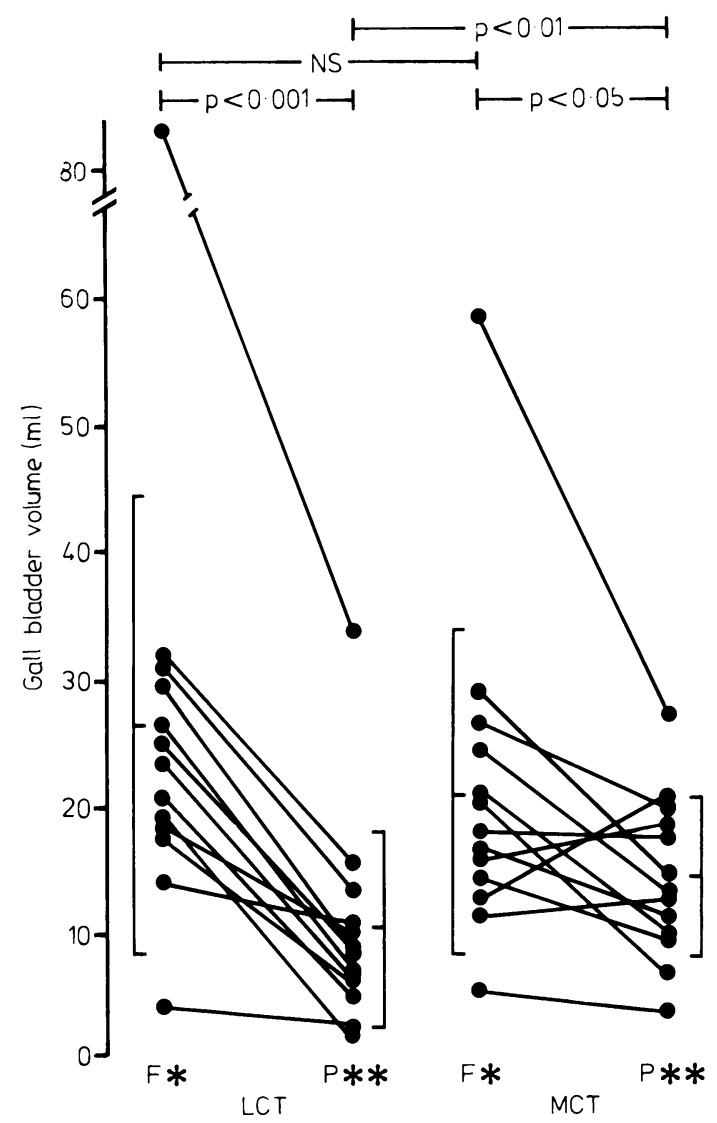

Fig. 1 Fasting $\left(F^{*}\right)$ and smallest postprandial $\left(P^{* *}\right)$ gall bladder volumes estimated by ultrasound in 13 normal subjects after ingestion of long chain triglyceride and medium chain triglyceride test meals. Lines indicate individual subjects. Vertical lines are mean $\pm I S D$.

confirmed fluoroscopically at the beginning and the end of each study. Twenty five centimetres proximal to the ileal aspiration point a plasma like solution (sodium $135 \mathrm{mmol} / \mathrm{l}$, potassium $5 \mathrm{mmol} / \mathrm{l}$, chloride $105 \mathrm{mmol} / \mathrm{l}$, bicarbonate $35 \mathrm{mmol} / \mathrm{l}$, osmolality 280 $\mathrm{mOsm} / \mathrm{kg}$ ) containing $10 \mu \mathrm{Ci} / \mathrm{l}$ of ${ }^{14} \mathrm{C}-\mathrm{PEG}$ as non-absorbable marker and $2 \mathrm{~g} / \mathrm{l}$ of PEG 4000 as carrier was infused at a rate of $0.3 \mathrm{ml} / \mathrm{min}$ using a Harvard peristaltic pump model 1203A (Southnatick, Mass). After a one hour equilibration period, fasting samples of jejunal and ileal contents were collected for two half hour periods using a syringe pump (perfusor MK IV, Braun AG Melsungen) set at an aspiration rate of $0.2 \mathrm{ml} / \mathrm{min}$. The volumes obtained from the jejunum and ileum in 30 minutes were between one and five mililitres 
(median: $3 \mathrm{ml}$ ). The volunteers then drank either the long chain triglyceride or the medium chain triglyceride test meal, which contained $150 \mathrm{mg}$ of phenol red (PR) as a non-absorbable marker. Half hour samples were continuously aspirated with the syringe pump from the jejunum and terminal ileum for eight periods. The test was repeated on the next day with the other meal in random order. Estimated gonadal irradiation at fluoroscopy was $50-70 \mathrm{mrad}$ and colonic radiation by infused ${ }^{+} \mathrm{C}$ was $70 \mathrm{mrad}$ per study.

Samples obtained were stored at $-20^{\circ} \mathrm{C}$ and estimations were made of phenol red by absorption at $560 \mathrm{~nm}$ in the sample adjusted to $\mathrm{pH} 9 \cdot 2,{ }^{17}$ of sodium by flame photometry and of total bile acid concentrations by enzymatic fluorimetric method ${ }^{18}$ using uncentrifuged specimens. Duplicate $100 \mu \mathrm{l}$ aliquots of each sample were added to $10 \mathrm{ml}$ of NE 260 scintillation cocktail (Nuclear Enterprises, Sighthill, Edinburgh) and counted on a liquid scintillation counter (Rack-Beta 1215, LKB-Wallac, Finland). A colour quench correction curve was made using a series of ileal aspirates to which were added a fixed amount of ${ }^{14} \mathrm{C}-\mathrm{PEG}$ and increasing amounts of phenol red within the range observed in the samples.

The volume of the meal as it passed the upper jejunum $(\mathrm{Vj})$ was estimated as:

$$
\mathrm{Vj}=\mathrm{Vm} \times[\mathrm{PRm}] \times[\mathrm{PRj}]^{-1}
$$

where $\mathrm{Vm}$ is the original volume of the meal $(300$ $\mathrm{ml}),[\mathrm{PRm}]$ the phenol red concentration in the meal $(0.5 \mathrm{mg} / \mathrm{ml})$ and $[\mathrm{PRj}]$ the maximum phenol red concentration observed in the jejunum postprandially. The volume of the postprandial jejunal contents calculated in this way is only an estimate of the minimal volume of fluid passing the jejunal aspiration port of the tube, but should reflect the relative dilution and transit of the two meals in the upper intestine. ${ }^{19}$

The ileal flowrate $\left(F_{i}\right)$ was calculated from the change in concentration of the infused marker $\left({ }^{14} \mathrm{C}-\mathrm{PEG}\right)$ according to the formula:

$$
F_{i}=F_{\text {in }} \times\left[P G_{\text {in }}\right] \times\left[P G_{\text {out }}\right]^{-1}
$$

where $F_{\text {in }}$ is the infusion rate $(0 \cdot 3 \mathrm{ml} / \mathrm{min}),\left[P G_{\text {in }}\right]$ the disintegrations per minute in the infusate and $\left[\mathrm{PEG}_{\text {out }}\right]$ the disintegrations per minute in the ileal sample. The above formula assumes that there was complete mixing of the infusate with the ileal contents and that the small volume $(18 \mathrm{ml} / \mathrm{h})$ of the infusate was completely absorbed from the terminal $25 \mathrm{~cm}$ of ileum. These assumptions are not entirely justified but, as discussed by Levitt and Bond ${ }^{20}$ the choice of a slow rate of marker infusion, though giving rise to possible inaccuracies during changing states, is most appropriate to the ileum where the volume of fluid is usually very small. Small bowel transit time was taken as the time interval between the first visible appearance of phenol red in the jejunal and ileal aspirates.

STATISTICAL ANALYSES

To compare the different local responses to the arrival of the two meals, variability of transit has been eliminated and the results were grouped to give parallel time concentration curves of phenol red. Subsequently, using probability graph paper, it was determined whether the frequency distribution of the results could be closely approximated by a normal distribution. ${ }^{21}$ Results with a normal distribution were tested for significance by analysis of variance appropriate to an experiment with repeated measures of a single factor, ${ }^{2}$ and results were expressed as mean $\pm 1 \mathrm{SD}$. Non-normally distributed results were expressed as median and range and differences examined by Wilcoxon's signed rank test.

\section{Results}

\section{GALL BLADDER}

The fasting gall bladder volumes before medium chain triglyceride $(21.2 \pm 12.5 \mathrm{ml})$ and long chain triglyceride meals $(26 \cdot 6 \pm 18 \cdot 0 \mathrm{ml})$ were not significantly different $\left(F_{1.12}=4 \cdot 13, p>0 \cdot 07\right)$. The minimum postprandial volume was significantly less than the fasting volume for both medium chain triglyceride meals $\left(14 \cdot 3 \pm 6 \cdot 0 \mathrm{ml}, \mathrm{F}_{1.12}=6 \cdot 16\right.$, $\mathrm{p}<0 \cdot 005)$ and long chain triglyceride meals $\left(10 \cdot 3 \pm 7.7 \mathrm{ml}, \quad \mathrm{F}_{1.12}=21.35, \quad \mathrm{p}<0.001\right)$. The minimum gall bladder volume observed after the long chain triglyceride meal, however, was significantly greater than that after the long chain triglyceride meal $\left(\mathrm{F}_{1.12}=9 \cdot 81, \mathrm{p}<0 \cdot 01\right)$. The mean reduction of gall bladder volume [(fasting-minimum postprandial volume/fasting volume $) \times 100]$ was greater after the long chain triglyceride meal $(59.0 \pm 17 \cdot 1 \%)$ than after the medium chain triglyceride meal $\left(23.4 \pm 33.6 \%, \quad F_{1.12}=12 \cdot 33\right.$, $\mathrm{p}<0 \cdot 005$ ).

\section{UPPER JEJUNUM}

After the ingestion of the long chain triglyceride meal the jejunal bile acid concentration rose significantly $\left(\mathrm{p}<0.001, \mathrm{~F}_{9.36}=4.64\right)$ (Fig. 2a), but was unchanged after the ingestion of the medium chain triglyceride test meal $\left(p=0 \cdot 2, F_{9.36}=1 \cdot 54\right)$. The differences between the jejunal bile acid concentration after the two meals were significant at one hour $\left(p<0.05, F_{1.4}=9.24\right), 1 \frac{1}{2}(p<0.01$, $\left.F_{1.4}=29 \cdot 80\right)$ and two hours $\left(p<0 \cdot 05, F_{1,4}=12.39\right)$ postprandially, although the estimated volumes of 

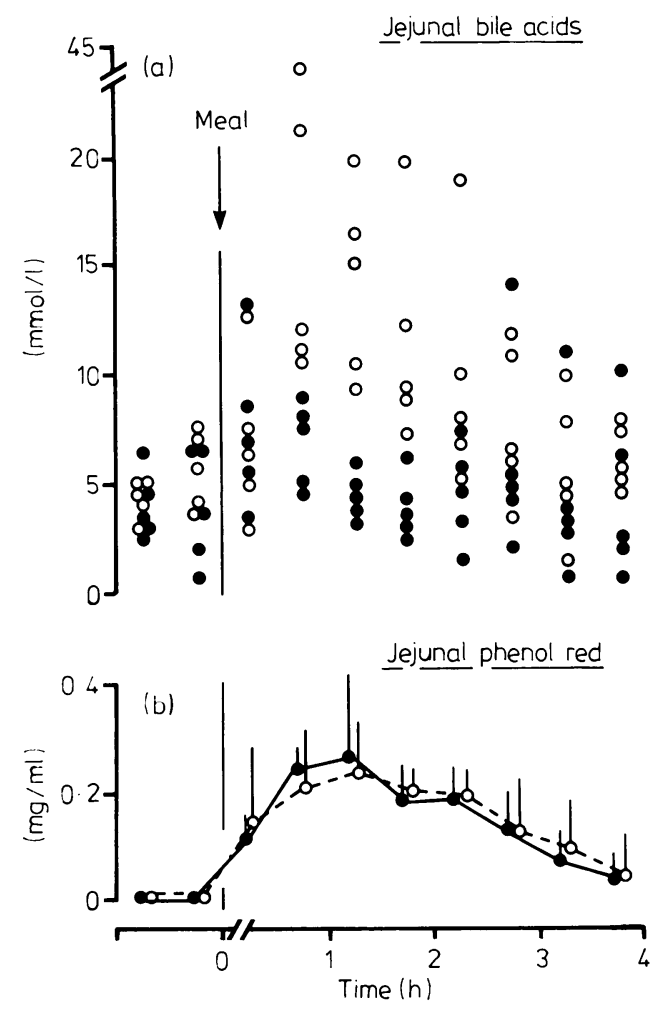

Fig. 2 (a) Jejunal bile acid concentrations in each half hour samples after medium chain triglyceride (O) and long chain triglyceride (O) meals. (b) Phenol Red concentrations (mean $\pm 1 S D$ ) in jejunal samples after medium chain triglyceride (-) and long chain triglyceride $(\bigcirc--\bigcirc)$ meals containing $0.5 \mathrm{mg} / \mathrm{ml}$ of phenol red.

$N B$ Variation of mouth to jejunum transit time between individuals and meals has been eliminated (see statistical analysis).

the postprandial jejunal contents were not significantly different $(540 \pm 150$ and $522 \pm 169 \mathrm{ml}$, medium chain triglyceride $v s$ long chain triglyceride, $\mathrm{p}=0 \cdot 80, \mathrm{~F}_{1.4}=0 \cdot(09)$. Furthermore, the meal marker (phenol red) concentration curves were almost identical (Fig. 2b), strongly suggesting that the two meals were being diluted in the upper jejunum to the same extent. Finally, there was no significant difference between the postprandial sodium concentrations after the long chain triglyceride meal $(106 \pm 15 \mathrm{mmol} / \mathrm{l})$ and those after the medium chain triglyceride test meal $\left(101 \pm 17 \mathrm{mmol} / \mathrm{l} \mathrm{F}_{1.78}=1 \cdot 70\right.$, $\mathrm{p}=0 \cdot 8$ ).

TERMINAL ILEUM

The small bowel transit time of phenol red was $66 \pm 12$ and $54 \pm 12$ minutes for the medium chain triglyceride and long chain triglyceride test meals respectively $\left(F_{1.4}=1, p=0 \cdot 4\right)$. As shown in Fig 3a, the ileal flow rate did not increase above the fasting value when the medium chain triglyceride meal marker (phenol red) reached the terminal ileum (Fig. 3b). The arrival of the long chain triglyceride meal marker (phenol red) in the terminal ileum, however, coincided with a consistent increase in the ileal flow rate which lasted for one hour (Fig. 3a). The postprandial ileal flow was $48 \cdot 5 \pm 12 \cdot 6$ and $123 \cdot 4 \pm 35 \cdot 2 \mathrm{ml} / \mathrm{h}$ for the medium chain triglyceride and long chain triglyceride test meals respectively $\left(F_{1.4}=28 \cdot 95, p<0 \cdot 01\right)$. The bile acid concentrations were higher after the long chain triglyceride meal $(1 \cdot 0,0 \cdot 5-30 \cdot 0 \mathrm{mmol} / \mathrm{l}$ median and range) than after the medium chain triglyceride meal $(0 \cdot 5 \quad 0 \cdot 2-1 \cdot 3$
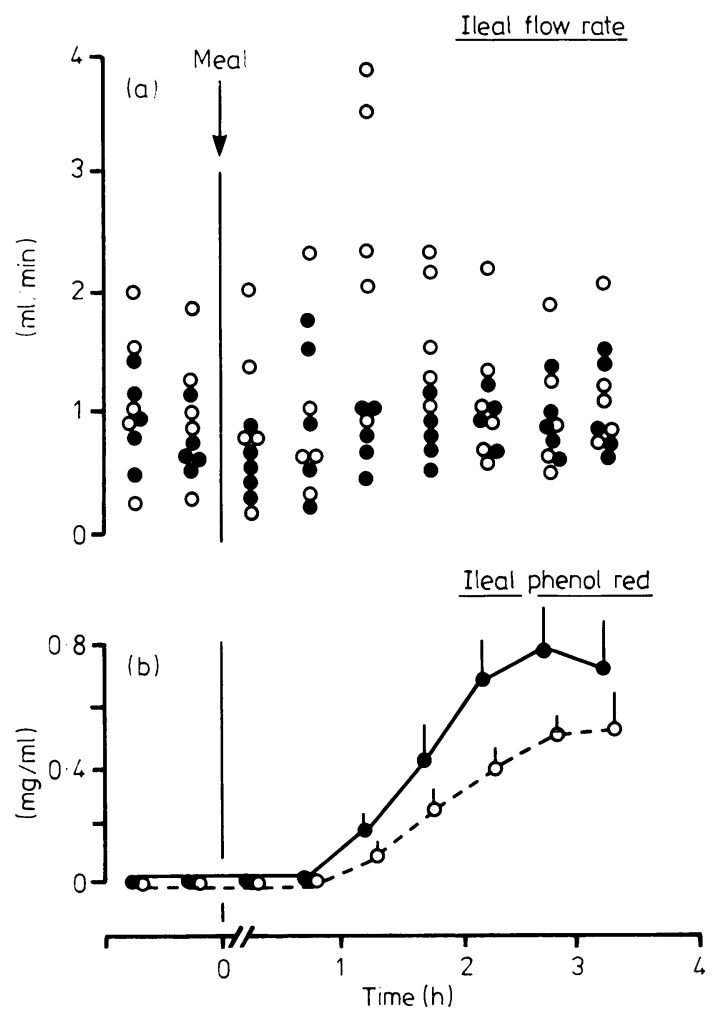

Fig. 3 (a) Ileal flow during each half hour period after medium chain triglyceride (O) and long chain triglyceride (O) meals. (b) Phenol Red concentrations (mean \pm ISD) in ileal samples after medium chain triglyceride (-) and long chain triglyceride $(\bigcirc---\bigcirc)$ meals containing $0.5 \mathrm{mg} / \mathrm{ml}$ of phenol red.

$N B$ Variation of mouth to ileum transit time between individuals and meals has been eliminated (see statistical analysis). 
$\mathrm{mmol} / \mathrm{l}$ ), but this difference was not statistically significant. The postprandial bile acid colonic input, however, was significantly higher after the long chain triglyceride meal $(0 \cdot 10,0.03-2.79 \mathrm{mmol} / \mathrm{h})$ than after the medium chain triglyceride meal $(0 \cdot 02$, $0 \cdot 01-0.04 \mathrm{mmol} / \mathrm{h})(\mathrm{p}<0 \cdot 05$, Wilcoxon's signed rank test). Though the postprandial sodium concentrations were not significantly different after the two meals $(139 \pm 11$ and $133 \pm 8 \mathrm{mmol} / \mathrm{l}$ long chain triglyceride $v s$ medium chain triglyceride, $\mathrm{p}=0 \cdot 07$ ), the total amount of sodium passing down the ileum postprandially was significantly higher after the long chain triglyceride meal $(17 \cdot 20 \pm 5 \cdot 30 \mathrm{mmol} / \mathrm{h})$ than after the medium chain triglyceride meal $(6 \cdot 40 \pm 1 \cdot 60$ $\mathrm{mmol} / \mathrm{h}, \mathrm{F}_{1.4}=26 \cdot 57, \mathrm{p}<0 \cdot 01$ ).

\section{Discussion}

This study has shown several important differences in the gut and gall bladder responses to medium chain triglyceride rich compared with long chain triglyceride rich liquid meals in normal man. We would attribute these differences to the nature of the fat ingested, although the meals differed in relation to ionic composition and osmolality, which might affect gastric emptying and intestinal transit. The dilution of the meal marker in the jejunum, the jejunal sodium concentrations and small bowel transit time, however, were not significantly different for the two meals and therefore differences in gastric emptying or transit cannot explain our results. It seems likely that the different effect of the two meals on the gall bladder is the key to the other phenomena.

Because the phenol red concentration curves in the jejunum indicate that the transit and dilution of the meals was similar, the greater concentration of bile acids in the duodenum after the long chain triglyceride is probably caused by a great input of bile acid into the duodenum. A more vigorous gall bladder emptying in response to long chain triglyceride can be inferred from the studies of Malagelada et $a l^{13}$ who perfused fatty acids through the human duodenum and noted that the bilirubin input increased with increasing chain length of the fatty acid infused. In the present study, medium chain triglyceride and long chain triglyceride rich meals clearly produced markedly different gall bladder responses. If gall bladder contraction is mainly influenced by cholecystokinin it seems likely that only a small release of cholecystokinin was triggered by the presence of medium chain triglyceride in the duodenum and further studies examining cholecystokinin response are now being undertaken.

From the estimated final volume of either meal in the jejunum of $540 \mathrm{ml}$ and the observed bile acid concentrations of 15.2 and $5.0 \mathrm{mmol} / \mathrm{l}$ (long chain triglyceride and medium chain triglyceride respectively), it may be estimated that an average of about 7.6 and $2.5 \mathrm{mmol}$ of bile acid were delivered into the duodenum after the long chain triglyceride and medium chain triglyceride meals respectively. If gall bladder bile after an overnight fast contains approximately $0.2 \mathrm{mmol} / \mathrm{ml}$ of bile acid, ${ }^{23}$ the observed gall bladder ejection of $16 \mathrm{ml}$ after one long chain triglyceride meal would contain $3.2 \mathrm{mmol}$ of bile acid and account for less than half of the bile acid delivered into the jejunum after the long chain triglyceride meal. The remainder is presumably derived from a stimulated hepatic choleresis. The amount of bile acid delivered into the upper jejunum after the medium chain triglyceride meal $(2.5 \mathrm{mmol})$, however, was close to that similarly estimated from the gall bladder ejection volume $(7 \mathrm{ml} \times 0.2 \mathrm{mmol} / \mathrm{ml}=1.4 \mathrm{mmol})$, suggesting that the medium chain triglyceride meal had a lesser effect on hepatic bile flow. These are indirect estimates, however, and it is possible that rapid absorption of bile acids in the upper jejunum ${ }^{2+}$ after the medium chain triglyceride meal has accentuated the difference in bile acid concentrations. The main differences in the test meals other than the lipid composition were that the medium chain triglyceride meal was of higher osmolality. This would tend to slow gastric emptying, but as Hunt and $\mathrm{Knox}^{25}$ have shown that gastric emptying is impaired by long chain triglyceride, it seems likely that these two effects have cancelled each other out and the two meals have entered the jejunum at approximately the same rate.

The phenol red concentration curves in the jejunum indicate that the transit and dilution of the meals was similar. Also the jejunal sodium concentrations were not significantly different for the two meals. It would seem, therefore, that differences in gastric emptying and/or transit cannot explain our results. The use of phenol red as a marker of meal transit and dilution, however, is subject to the same limitations as the use of any single phase marker. Lipid soluble markers may not be appropriate as they have been validated only for long chain triglyceride and could be more difficult to interpret when mixtures of long chain triglyceride and medium chain triglyceride enter the duodenum, becoming richer in long chain triglyceride after the rapid absorption of the medium chain triglyceride. Our overall conclusions contrasting the effects on normal gut function of long chain triglyceride- and medium chain triglyceride-containing diets appear to be supported by the observations of Hofmann and Poley ${ }^{5}$ on patients with ileal resection that lower 
hepatic bile synthesis and faecal bile acid excretion occur in subjects taking medium chain triglyceride compared with long chain triglyceride diets. Although they found that the small intestine absorbs more than $95 \%$ of the amount of bile acids entering the upper jejunum after the ingestion of either meal, we have shown the bile acid colonic input to be significantly higher after the long chain triglyceride meal. This could also explain the increased ileal flow of fluid and sodium into the colon after the long chain triglyceride meal because conjugated dihydroxy bile acids impair the absorption of salt and water by the human small intestine. ${ }^{9}{ }^{10}$

This increased flow of water and bile acids into the colon after a long chain triglyceride meal is likely to produce diarrhoea in patients with either small or large gut disease. In small gut disease, the amounts of fluid and bile acids entering the colon are likely to be greater if small gut absorption is impaired. In colonic disease, the capacity of the colon to absorb water is already impaired and could be reversed to secretion if concentrations of luminal bile acids were increased. These phenomena may account for the observation that restriction of dietary fat (long chain triglyceride) intake is often of great benefit to patients with diarrhoea after small bowel resections of various types.

Finally, dietary fat chain length may be of relevance to the incidence of colonic cancer. A high incidence of colon cancer has been related epidemiologically to a high intake of dietary fat ${ }^{26}$ and high faecal bile acid excretion 2728 and possibly to an increased colonic input and bacterial degradation of bile acids after cholecystectomy. ${ }^{29} 30$ Our findings suggest that in studies relating fat intake to bile acid excretion or turnover, the chain length, as well as the total amount, of dietary fat is also worthy of attention.

The authors are indebted to Mr Y Qureshi for his technical assistance and to Miss Kate Teasdale for her secretarial assistance. Drs Murphy and Ladas were supported by the Wellcome Foundation.

\section{References}

1 Winawer SJ, Broitman SA, Wolochow DA et al. Successful management of massive small-bowel resection based on assessment of absorption defects and nutritional needs. $N$ Engl J Med 1966; 274: 72-7.

2 Zurier RB, Campbell RG, Hashin SA et al. Use of medium-chain triglyceride in management of patients with massive resection of the small intestine. $N$ Engl $J$ Med 1966; 274: 490-6.

3 Bochenek W, Rodgers JB, Balint J. Effects of changes in dietary lipids on intestinal fluid loss in the shortbowel syndrome. Ann Intern Med 1970; 72: 205-13.

4 Greenberger NJ, Rupert RD, Tzagournis M. Use of medium chain triglycerides on intestinal fluid loss in the short bowel syndrome. Ann Intern Med 1970; 72: 205-13.

5 Hofmann AF, Poley JR. Role of bile acid malabsorption in pathogenesis of diarrhoea and steatorrhoea in patients with ileal resection. I. Response to cholestyramine or replacement of dietary long chain triglyceride. Gastroenterology 1972; 62: 918-34.

6 Holt PR. Medium chain tryglycerides. Their absorption, metabolism and clinical applications. In: Glass BGJ, ed. Progress in gastroenterology New York: Grune, 1968: 277-98.

7 Greenberger NJ, Skillman TG. Medium chain triglycerides: Physiologic considerations and clinical implications. N Engl J Med 1969; 280: 1045-58.

8 Hofmann AF. Intraluminal factors in the absorption of glycerides. In: Senior JR, ed. Medium chain triglycerides. Philadelphia: University of Pennsylvania Press, 1968: 9-19.

9 Wingate DL, Krag E, Mekhjian HS et al. Relationships between ion and water movement in the human jejunum, ileum and colon during perfusion with bile acids. Clin Sci Mol Med 1973; 45: 593-606.

10 Krag E, Phillips SF. Effect of free and conjugated bile acids on net water, electrolyte and glucose movement in the perfused human ileum. J Lab Clin Med 1974; 83: 947-56.

11 Redinger RN, Hermann AH, Small DM. Primate biliary physiology. X. Effects of diet and fasting on biliary lipid secretion and relative composition and bile salt metabolism in the rhesus monkey. Gastroenterology 1973; 64: 610-21.

12 Ladas S, Isaacs PET, Qureshi Y et al. Effect of long and medium chain tryglyceride meals on human ileal flow rate and luminal bile acid concentrations. [Abstract] Clin Sci 1982; 61: 39P.

13 Malagelada J-R, DiMagno EP, Summerskill WHJ et al. Regulations of pancreatic and gall bladder function by intraluminal fatty acids and bile acids in man. J Clin Invest 1976; 58: 493-9.

14 Braverman DZ, Johnson ML, Kern F Jr. Effects of pregnancy and contraceptive steroids on gallbladder function. $N$ Engl J Med 1980; 302: 362-4.

15 Everson GT Braverman DZ, Johnson ML et al. A critical evaluation of real-time ultrasonography for the study of gallbladder volume and contraction. Gastroenterology 1980. 79: 40-6.

16 Wiener I, Inoue $\mathrm{K}$, Fagan $\mathrm{ChJ}$ et al. Release of Cholecystokinin in man: correlation of blood levels with gallbladder contraction. Ann Surg 1981; 194: 321-5.

17 Schedl HP, Clifton JA. Small intestinal absorption of steroids. Gastroenterology 1961; 41: 491-9.

18 Talalay P. Enzymatic analysis of steroid hormones. Biochem Anal 1960; 8: 119-43.

19 Fordtran JS, Locklear TW. Ionic constituents and osmolality of gastric and small-intestinal fluids after eating. Am J Dig Dis 1966; 11: 503-21. 
20 Levitt MD, Bond J. Use of the constant perfusion technique in the nonsteady state. Gastroenterology 1977; 73: 1450-4.

21 Spiegel MR. Theory and problems of statistics. New York: McGraw-Hill, 1961.

22 Winer BJ. Statistical principles in experimental design. Tokyo: McGraw-Hill, Kogakusha, 1971.

23 Wheeler HO. The concentrating function of the gall bladder. Am J Med 1971; 51: 588-95.

24 McClintock C, Umstetter C, Shiau Y-F. Reassessing the role of the jejunum in bile salt absorpiton. [Abstract] Gastroenterology 1981; 80: 1227.

25 Hunt JN, Knox MT. An effect of fatty acid chain length on gastric emptying. J Physiol (Lond) 1968; 194: 327-36.
26 Wynder EL, Shigematsu T. Environmental factor of cancer of the colon and rectum. Cancer 1967; 20: 1520-61.

27 Aries VC, Crowther JS, Drasar BS et al. Bacteria and the aetiology of cancer of the large bowel. Gut 1969; 10: $334-5$.

28 Reddy BS, Wynder EL. Large bowel carcinogenesis: fecal constituents of populations with diverse incidence rates of colon cancer. J Natl Cancer Inst 1973; 50: 1439-42.

29 Linos DA, Beard CM, O'Fallon et al. Cholecystectomy and carcinoma of the colon. Lancet 1981; 2: 379-81.

30 Vernick LJ, Kuller LH. Cholecystectomy and rightsided colon cancer: an epidemiology study. Lancet 1981; 2: 381-3. 